\title{
Manganese-Induced Effects on Calcium Homeostasis of the Neurons in Hyline Cocks
}

\author{
Xiaofei Liu, Lirong Jing, Lv Xue, Yongqiang Ma, Huanan Guan, Wang Wei \\ College of Food Engineering, Harbin University of Commerce, Harbin 150076, China \\ email: liuxiaofei72@163.com
}

Keywords: Manganese; Calcium Homeostasis; Neurons; Cocks

\begin{abstract}
Manganese (Mn) is known to be essential for maintaining the proper function and regulation of many biochemical. To investigate the toxicity of Mn on bird brains, 50-day-old cocks were fed either a commercial diet or a Mn-supplemented diet. the following were determined: $\mathrm{Mn}$ concentration, $\mathrm{Ca} 2+$ concentration, the expression of Calmodulin (CaM) and Calcineurin (CaN) gene. Exposure to Mn significantly lowered expression of CaM and $\mathrm{CaN}$ gene. However, Mn was accumulated in brain and the $\mathrm{Ca} 2+$ concentration was increased. These findings suggested that $\mathrm{Mn}$ exposure resulted in the damage of cock cerebral tissue by altering calcium homeostasis, which are possible underlying neurotoxicity mechanisms.
\end{abstract}

\section{Introduction}

Manganese (Mn) is a naturally occurring trace metal commonly found in the environment. While exposure to higher Mn concentrations is harmful to organisms [1]. It is a common finding that a wild bird is affected by Mn toxicity. Piscivorous birds, such as herons and egrets, are suitable indicator organisms of environmental pollution in aquatic systems [2]. These birds belong to the upper trophic chain levels of these ecosystems; as a consequence, they bioaccumulate hydrophobic contaminants.

A regulated rise in the intracellular $\mathrm{Ca}^{2+}$ concentrations $\left(\left[\mathrm{Ca}^{2+}\right] \mathrm{i}\right)$ is a key signal in neurons, and can trigger many physio-pathological events; but an unregulated elevation in $\left[\mathrm{Ca}^{2+}\right]$ i can alter cell viability. Recent evidence indicated that neuronal $\mathrm{Ca}^{2+}$ signaling was abnormal in many neurodegenerative disorders [3]. Calmodulin (CaM) is a prototypic EF-hand calcium-binding protein that acts by sensing calcium levels and binding to target proteins in a regulatory manner [4]. Although CaM is best characterized by its ability to specifically bind $\mathrm{Ca}^{2+}$, a number of studies have indicated that in fact it can also be activated by other metal ions. CaM or a CaM-like protein may be associated with the Mn-dependent adenylyl cyclase.

These previous studies led us to investigate Mn-induced brain toxicity in bird, especially involving the concentrations of $\mathrm{CaM}$ and Calcineurin $(\mathrm{CaN})$. The aim of this study was to provide a better understanding of Mn-induced cerebral toxicity in birds using the male Hyline cocks as a model.

\section{Materials and methods}

All procedures used in this research were approved by the Institutional Animal Care and Use Committee of Northeast Agricultural University. The method according to Liu et al [5].

Mn content were determinated. The samples of cerebral tissue were cut into small pieces and baked at $110{ }^{\circ} \mathrm{C}$ for $12 \mathrm{~h}$. After digestion, the samples were adjusted to $10 \mathrm{~mL}$ by $0.5 \% \mathrm{HNO}_{3}$ and characterized by flame atomic absorption spectrometry (FAAS) [6]. $\left[\mathrm{Ca}^{2+}\right]_{\mathrm{i}}$ assay was performed by a method described previously [7]. $\left[\mathrm{Ca}^{2+}\right]_{\mathrm{i}}=\mathrm{K}_{\mathrm{d}}\left[\left(\mathrm{R}-\mathrm{R}_{\min }\right) /\left(\mathrm{R}_{\max }-\mathrm{R}\right)\right] \times\left(\mathrm{S}_{\mathrm{f} 380} / \mathrm{S}_{\mathrm{b} 380}\right)$. The present experiments were carried out at $\mathrm{pH} 7.4$ and a temperature of $37{ }^{\circ} \mathrm{C}$. A $K_{d}$-value of $224 \mathrm{nM}$ was used. Quantification of CaM and CaN mRNA were measured.Total RNA was isolated from brain samples. Quantitative RT-PCR (qPCR) was used to detect the expression of CaM, CaN and $\beta$-actin gene in brain tissues. The method according to Liu et al [5].

All statistical analyses were performed 
using SPSS procedures (SPSS Inc., Chicago, IL, USA). One-way ANOVA was used to identify significant values $(p<0.05)$. Differences between means were assessed using Tukey's honestly significant difference test for post hoc multiple comparisons.

\section{Results}

The Mn content in the brain was shown in Fig.1. The results revealed that the Mn content appeared a dose and time-dependent fashion. There was an increasing tendency for $\mathrm{Mn}$ in $\mathrm{H}$ group compared to that of controls $(P<0.01)$. The Mn content in the M group at 30 and $60 \mathrm{~d}$ showed significant differences $(P<0.05)$. Compared with the corresponding control group, the Mn content in $\mathrm{H}$ group increased significantly $(P<0.01)$ at each time point.

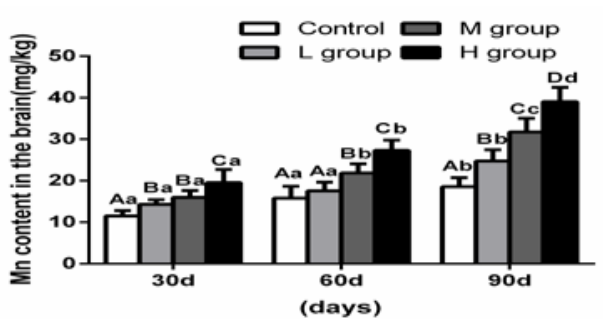

Fig. 1 Content of Mn in the brain

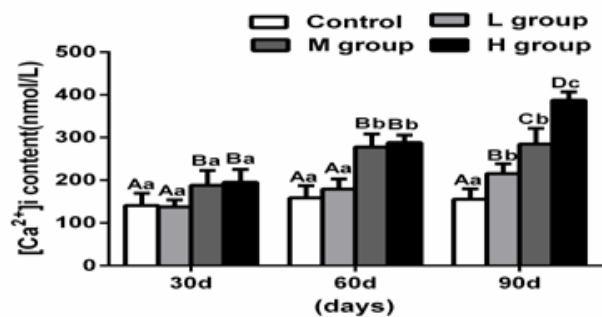

Fig. 2 Content of $\left[\mathrm{Ca}^{2+}\right] \mathrm{i}$ in the brain.

Note: Bars represent the mean \pm standard deviation ( $n=30$ /group). Bars with different uppercase letters are statistically significantly different in the same group. Bars with different lowercase letters are statistically significantly different at the same concentrations of dietary $\mathrm{Mn}$.

The effects of different concentrations of dietary $\mathrm{Mn}$ on the intracellular $\left[\mathrm{Ca}^{2+}\right]_{\mathrm{i}}$ were shown in Fig.2. The intracellular $\left[\mathrm{Ca}^{2+}\right]_{\mathrm{i}}$ in the cerebrum nerve cell was dose- and time-dependent and the values for all experimental groups increased daily compare to the control group values $(p<0.05)$, except for that in the $\mathrm{L}$ group at $30 \mathrm{~d}$. At the same experiment time point, intracellular $\left[\mathrm{Ca}^{2+}\right]_{\mathrm{I}}$ at $30 \mathrm{~d}$ decreased firstly, then increased with the Mn experiment dose. However, intracellular $\left[\mathrm{Ca}^{2+}\right]_{\mathrm{I}}$ at 60 and $90 \mathrm{~d}$ presented significantly ascend tendency. Notably, the intracellular $\left[\mathrm{Ca}^{2+}\right]_{\mathrm{i}}$ in the $\mathrm{H}$ group at $60 \mathrm{~d}$ and $90 \mathrm{~d}$ increased obviously by $62.9 \%$ and 1.49 times, respectively, compared with the control group.

The effects of different concentrations of dietary Mn on the mRNA levels of the CaM gene in the brain were shown in Fig.3A. The results showed that the mRNA levels of CaM gene appeared a dose and time-dependent fashion. On each experiment time point the mRNA levels of CaM in the $\mathrm{H}$ group decreased remarkably to $0.78,0.63$ and 0.44 times, respectively, compared with the corresponding control groups $(P<0.01)$. The effects of different concentrations of dietary Mn on the mRNA levels of the CaN gene were shown in Fig.3B. The results showed that the mRNA levels of CaN gene presented a descend trend, except for that at $30 \mathrm{~d}$. On 60 and $90 \mathrm{~d}$ the mRNA levels of $\mathrm{CaN}$ in the $\mathrm{H}$ group decreased remarkably to 0.67 and 0.41 times, respectively, compared with the corresponding control groups $(P<0.05)$.

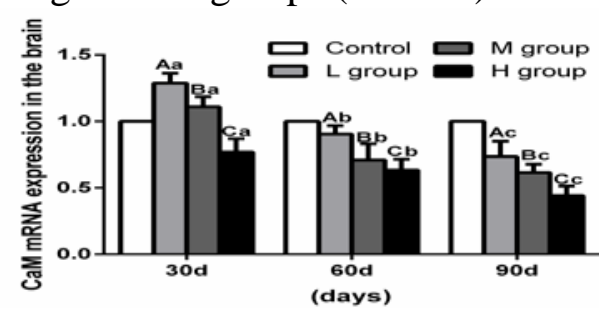

A the level of CaM

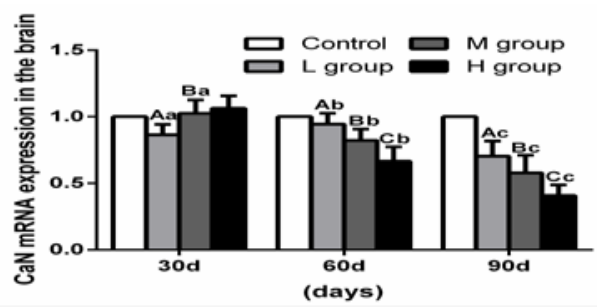

B the level of CaN

Fig. 3 Effects of Mn poisoning on the levels of CaM mRNA and CaN mRNA. Note: The meaning of bars is common to Fig.1.

\section{Discussion}

In this study, a model of Mn poisoning was established to study the effects of dietary Mn on brain damage in cocks. Our results revealed that the Mn contents in brain increased gradually with 
the Mn experiment dose. It was suggested that Mn had probably crossed the blood-brain barrier, and high dietary $\mathrm{Mn}$ led to its accumulation in the brain of cocks.Intramitochondrial $\mathrm{Ca}^{2+}$ may increase the rate of ATP production by several fold [8] by binding a series of $\mathrm{Ca}^{2+}$-sensitive sites in the metabolic pathways that have been shown by metabolic control theory to control the rate of this process. However, a rise in $\left[\mathrm{Ca}^{2+}\right] \mathrm{i}$ in Mn-treated neuronal cell as a result of the release of $\mathrm{Ca}^{2+}$ from intracellular stores and the inhibition of $\mathrm{Ca}^{2+}$ efflux is a necessary part of the mechanism, which was observed in the present study.

Much of $\mathrm{Ca}^{2+}$ signaling is mediated through the calcium-binding protein known as CaM. A number of proteins help regulate these pathways through their interaction with CaM [9]. Calcium/calmodulin $\left(\mathrm{Ca}^{2+} / \mathrm{CaM}\right)$-dependent protein phosphorylation has been implicated in various cellular processes [10]. CaM is able to interact with a plethora of target proteins having diverse functions. CaN is a calcium- and calmodulin-dependent protein serine/threonine phosphate which is critical for several important cellular processes. Wei et al[11] reported that X-band electron spin resonance spectroscopy was used to investigate the binding of $\mathrm{Mn}^{2+}$ to the apo-forms of calcineurin and its A and B subunits. These results, based on direct spectral measurements of the metal ligand, demonstrated that $\mathrm{Mn}^{2+}$ binds to both free subunits of $\mathrm{CaN}$ in a manner distinct from binding to the holoenzyme. Also, the data suggest that conformational changes occur upon heterodimer formation and association of the holoenzyme with the regulatory protein CaM. Wu et al [12] reported that the active CaM concentration of the striatum tissues exposed to $10 \mathrm{mg} / \mathrm{kg}$ of $\mathrm{Mn}$ elevated obviously in comparison with the control group, and the active CaM concentration of the striatum tissue decreased as dose of $\mathrm{Mn}$ increased. Similar to this result, in the present study, Mn treatment significantly inhabited the expression of CaM gene and CaN gene in cerebral tissue.

\section{Conclusion}

$\mathrm{Mn}$ is toxic to brain cells of bird. The $\mathrm{Ca}^{2+}$ concentration was increased and the expression of $\mathrm{CaM}$ and $\mathrm{CaN}$ gene was decreased along with $\mathrm{Mn}$ diet. The apoptosis of the neurons induced by excessive $\mathrm{Mn}$ affect calcium homeostasis. Mn poisoning caused brain intracellular $\mathrm{Ca}^{2+}$ overload and calcium dyshomeostasis possibly be one of the mechanisms that induce the central nervous system damage.

\section{Acknowledgement}

In this paper, the research was sponsored by the Scientific Research Fund of Heilongjiang Provincial Education Department(Project No. 12541189).

\section{References}

[1] Chua AC, Morgan EH. Effects of iron deficiency and iron overload on manganese uptake and deposition in the brain and other organs of the rat[J]. Biol Trace Elem Res,1996.55: 39-54.

[2] Sakellarides TM. Accumulation profiles of persistent organochlorines in liver and fat tissues of various waterbird species from Creece[J]. Chemosphere, 2006.63: 1392-1409.

[3] Bezprozvanny I. Calcium signaling and neurodegenerative diseases[J]. Trends Mol Med, 2009.15(3): 89-100.

[4] Kursula P, Majava V. A structural insight into lead neurotoxicity and calmodulin activation by heavy metals[J]. Acta Cryst, 2007.63: 653-656.

[5]Liu XF, Li ZP, Han CR, et al. Effects of dietary manganese on $\mathrm{Cu}, \mathrm{Fe}, \mathrm{Zn}, \mathrm{Ca}$, IL-1 $\beta$, and IL-2 changes of immune Organs in cocks[J]. Biol Trace Elem Res. 2012.148(3): 336-44.

[6]AL-Gahr MA, Almussali MS. Microelement content of locally produced and imported wheat grains in Yemen[J]. E-Journal of Chemistry, 2008.5(4): 838--843. 
[7]Grynkiewicz G, Poenie M, Tsien RY. A new generation of $\mathrm{Ca}^{2+}$ indicators with greatly improved fluorescence properties[J]. J Biol Chem, 1985. 260(6): 3440-50.

[8]Kaleka KS, Petersen AN, Florence MA, et al. Pull-down of calmodulin-binding proteins[J]. J Vis Exp, 2012.23(59): doi:10.3791/3502.

[9]Jeong JC, Shin D. Isolation and characterization of anovel calcium/calmodulin-dependent protein kinase, AtCK, from Arabidopsis[J]. Mol. Cell, 2007.24(2): 276-82.

[10]Hoeflich KP, Ikura M. Calmodulin in action: diversity in target recognition and activation mechanisms[J]. Cell, 2002.108: 739-742.

[11]Wei Q, Xiao F, Lu J, Zhou J. ESR study on calcineurin[J]. Sci China B, 1995.38(9): 1117-22.

[12]Wu SL, Guo SC, Qin X, et al. Effect of taurine on active calmodulin content of the striatum tissue in manganese expgb osed rats[J]. Ind Hlth Occup Dis, 2008.34(2): 65-67 